\title{
Investigating Differences in Wiki-based Collaborative Activities between Student Engagement Profiles in CS1
}

\author{
Adam Eck and Leen-Kiat Soh \\ Department of Computer Science and Engineering \\ University of Nebraska-Lincoln \\ \{aeck, lksoh\}@cse.unl.edu
}

\author{
Duane F. Shell \\ Department of Educational Psychology \\ University of Nebraska-Lincoln \\ dshell2@unl.edu
}

\begin{abstract}
Introductory computer science courses are being increasingly taught using technology-mediated instruction and e-learning environments. The software and technology in such courses could benefit from the use of student models to inform and guide customized support tailored to the needs of individual students. In this paper, we investigate how student motivated engagement profiles developed in educational research can be used as such models to predict student behaviors. These models are advantageous over those learned directly from observing individual students, as they rely on different data that can be available a priori before students use the technology. Using tracked behaviors of 249 students from 7 CS1 courses over the span of 3 semesters, we discover that students with different engagement profiles indeed behave differently in an online, wiki-based CSCL system while performing collaborative creative thinking exercises, and the differences between students are primarily as expected based on the differences in the profiles. Thus, such profiles could be useful as student models for providing customized support in e-learning environments in CS1 courses.
\end{abstract}

\section{Keywords}

Computer Supported, Collaborative Learning; Creative Thinking; Student Modeling; Wiki; CS1

\section{INTRODUCTION}

Computer science 1 (CS1) courses are being increasingly taught using technology-mediated instruction and/or e-learning environments. For example, CS1 courses have used intelligent tutoring systems [1, 19], computer-supported, collaborative learning (CSCL) systems [4], interactive program visualizations [17] and interactions in e-books [5]. Reported benefits of these technology-mediated instruction and e-learning environments for CS1 courses include more flexibility and broader reach to offer education to a wider range of students, the ability to scale to larger classrooms such as Massively Open Online Courses (MOOCs) [2], and increased student engagement.

Furthermore, increased use of technology to deliver CS1 courses offers the capability to observe and record information about student behaviors during the learning process through passively mon-

Permission to make digital or hard copies of all or part of this work for personal or classroom use is granted without fee provided that copies are not made or distributed for profit or commercial advantage and that copies bear this notice and the full citation on the first page. Copyrights for components of this work owned by others than ACM must be honored. Abstracting with credit is permitted. To copy otherwise, or republish, to post on servers or to redistribute to lists, requires prior specific permission and/or a fee. Request permissions from Permissions@acm.org.

SIGCSE '16, March 02-05, 2016, Memphis, TN, USA C 2016 ACM. ISBN $978-1-4503-3685-7 / 16 / 03 \ldots \$ 15.00$

DOI: http://dx.doi.org/10.1145/2839509.2844615 itoring how students interact with the technology used for class instruction and assignments. This information can then be used to help educators better understand how students are learning within a course and aid in student evaluation. Combined with models of student behavior, the technology can also be automatically guided to adapt to observed student behaviors and provide customized instruction to individuals and groups in order to more efficiently and effectively improve student knowledge. That is, understanding and modeling student learning behaviors could help support and improve technology-mediated instruction or e-learning environments such as CSCL and MOOCs [2].

Indeed, machine learning and data mining and analysis have been applied to cluster student behaviors and model cognitive styles in e-learning environments in order to adapt learning behaviors, e.g., $[3,7,8,9]$. In particular, Desmarais \& Baker [3] advocated the importance of constructing models of learner behavior, such as through Bayesian Networks, based on observed activities and other key constructs such as learner motivation, emotional and attentional state, metacognition and self-regulated learning. However, these studies constructed their models based on observing student behavior in the e-learning environment, instead of considering student models based solely on learner motivation, metacognition, and self-regulation that might be knowable through surveys or self-reports a priori to or separately from the students' use of technology within the learning environment. These studies also have not necessarily focused on CS education or introductory programming courses.

In this paper, we begin to bridge this gap in the literature by investigating how student motivated engagement profiles that have been identified in educational research $[11,15,16]$ relate in practice to student learning behaviors in technology-mediated instruction and e-learning environments within CS1 courses. That is, instead of developing empirical models based on observed behavior, we are instead interested in comparing how students with different motivated engagement profiles identified from student survey questionnaires actually behaved, as recorded by their interactions with technology during course work. This comparison is important because provided that students indeed behave as predicted, then we have evidence that engagement profiles, which are potentially identifiable prior to learning activities, can be used as models and predictors of student learning behaviors in ways that may guide the customization of e-learning to individual student's motivation and engagement needs in CS courses.

In particular, this paper addresses the following research questions:

RQ1: Do CS1 students with different engagement profiles behave differently in an online CSCL system? 
RQ2: Do different behaviors exhibited by CS1 students in an online CSCL system match the behaviors that would be expected based on their engagement profile?

To answer these questions, we considered data from 249 students within 7 CS1 courses spanning 3 semesters (Fall 2012, Spring 2013, Fall 2013). Each of these students completed a survey identifying their goal orientation, perceived benefits of the course, and emotional/affective states in order to match students to different learning profiles. During their course, students performed groupbased creative thinking exercises using CSCL wiki software as part of their CS1 education, where exercises were created on wiki pages. Through their use of the CSCL wiki software, we tracked when students performed different actions needed to complete their assignments. Based on this tracked data, our investigation compared how often each type of action was performed by each student, and we compared the trends in these measures to what should be expected based on their profiles.

Through our investigation, we discovered that: (1) observed student behaviors differed for different engagement profiles, indicating that engagement profiles indeed affect student behavior, and (2) the differences between behaviors for the different profiles largely matched what was predicted by the educational theory behind the engagement profiles, indicating that such profiles are indeed predictors of student behavior in technology-mediated instruction and e-learning environments. This implies that a CSCL tool such as the wiki platform considered in our study can effectively model student learning profiles and have the potential to inform instruction and support student learning at real-time, with important possible benefits for CS1 (and other) courses.

\section{BACKGROUND}

\subsection{Motivated Engagement Profiles}

Prior research has identified five distinct motivated engagement profiles among college students $[11,15,16]$. These are:

1. A strategic learner who demonstrates a high level of motivation, metacognition, and engagement and aspires to master course content and achieve highly in the classroom;

2. A knowledge builder who is motivated to learn course content for their personal growth, but does not necessarily exhibit high levels of active engagement or metacognitive strategies;

3. A surface learner who is motivated only by the utility value of the course and engages at superficial rather than deep learning;

4. An apathetic learner who demonstrates low levels of motivation and engagement; and

5. A learned helpless student who is motivated to engage but ineffective in their learning and strategies, eventually causing them to lose motivation and begin to disengage.

Recent studies have found these five profiles among undergraduate CS, engineering, and other STEM students taking CS1 courses $[6,11,16]$. In these CS courses, students adopting the strategic and knowledge builder profiles have higher course achievement and learning $[11,16]$.

\subsection{Creative Thinking Exercises}

The CS1 courses considered in this study incorporated both computational thinking and creative thinking into CS1 courses to better prepare students to be flexible and resourceful problem solvers, interdisciplinary collaborators, and skilled practitioners of both logic and creativity [18]. This was accomplished through creative thinking exercises designed to challenge established thinking and behavior patterns. These creative thinking exercises consist of a set of activities, some "light bulbs" to help students connect these activities to a given topic, as well as a set of analysis and reflection questions for students to discuss and respond to.

For example, one collaborative creative thinking exercise asked students to tell a story collaboratively. Each team of students was assigned a story, with each team member being responsible for a chapter. We provided each chapter with its first and last sentences and required the student responsible for that chapter to fill in 100200 words between those two sentences. Additionally, the last sentence of the first chapter is the same as the first sentence of the second chapter, the last sentence of the second chapter was the same as the first sentence of the third chapter, and so on. After one week of working on each chapter individually, team members were then required to combine all chapters into a story and address all inconsistencies in the chapter in the second week. Through this process, students learned about logical reasoning, "debugging", and creative problem solving as well.

The other creative thinking exercises included Everyday Object where students were asked to describe an everyday object such as a ball-point pen as if the object had never been invented, Cipher where students were asked to devise encoding rules to map alphabet letters to numbers and to decipher other teams' code, Exploring where students were asked to visit a location on campus and record what they observed through different senses (in addition to sight), and so on.

\subsection{Online Wiki-based CSCL System}

To collaborate and complete their creative thinking projects, students used an online, wiki-based CSCL platform [4]. This platform was selected because it supports flexible group assignments to setup collaborative work; asynchronous communications between students working together on collaborative writing assignments to promote coordination and cooperation between group members; revision tracking on wiki pages so that students can better understand how content changes over time based on the contributions of different group members; and fine grained tracking of student activities, including who performed an action, what page the action was performed on, when the action occurred, and what type of action was performed.

Using the wiki, each student could perform three key actions toward completing their collaborative creative thinking exercises:

1. Viewing wiki pages, including the assignment instructions for each exercise, exemplar examples of solutions to the exercises from past semesters, and the student's group's solutions to the exercises.

2. Editing wiki pages, especially by making changes to the student's group's solution to each exercise, building upon and revising the past contributions of team members, and

3. Commenting on wiki pages within threaded discussions, leaving messages for group members to read and respond to, in order to discuss and coordinate the students' activities towards completing the creative thinking exercises.

Other actions were also possible within the wiki (e.g., searching for pages, viewing pages intelligently recommended by the system related to the student's current page, rating pages, etc.). However, within the context of the creative thinking exercises, the three key actions (view, edit, comment) outlined above constitute the main behaviors required of students to complete the exercises. Thus, they were the focus of student behavior in this study.

\section{DATA}

To better understand the relationship between (1) student motivated engagement profiles and (2) student behaviors during technol- 
Table 1: Summary of Students

Note: Other indicates class standing not fitting into the standard categories (e.g., non-traditional or graduate students)

\begin{tabular}{c|c}
\hline Total & $249(100 \%)$ \\
\hline Males & $213(85.5 \%)$ \\
\hline Female & $36(14.5 \%)$ \\
\hline Freshman & $117(47.0 \%)$ \\
\hline Sophomore & $79(31.7 \%)$ \\
\hline Juniors & $34(13.7 \%)$ \\
\hline Seniors & $16(6.4 \%)$ \\
\hline Other & $3(1.2 \%)$ \\
\hline \hline CS Majors & $61(24.5 \%)$ \\
\hline CS Non-majors & $188(75.5 \%)$
\end{tabular}

Table 2: Motivated Engagement Profile Statistics Engagement Profiles Number of Students

\begin{tabular}{r|c}
\hline \hline Strategic & $61(24.5 \%)$ \\
\hline Knowledge Builders & $64(25.7 \%)$ \\
\hline Surface & $51(20.5 \%)$ \\
\hline Apathetic & $39(15.7 \%)$ \\
\hline Learned Helpless & $34(13.7 \%)$
\end{tabular}

ogy-mediated instruction and e-learning environments in our CS1 courses, we investigated the behaviors of students completing the creative thinking exercises within the aforementioned CSCL system. In this section, we describe the data collected and analyzed within our investigation.

The creative thinking exercises described in Section 2.2 were deployed to several CS1 courses including a course designed for engineering students and another for CS majors. For this study, we considered data collected from 7 classes at the University of Nebraska-Lincoln across 3 semesters (Fall 2012, Spring 2013, Fall 2013), with a total of 249 students consenting to the use of their engagement profile survey results (described below) and tracked behaviors. Summary statistics for these students are provided in Table 1. In spite of the fact that we considered students from introductory (CS1) courses, the students had a wide variety of experience in school and a range of majors. Thus, our results should generalize to a wide range of potential CS1 students.

Profiles for this study population were derived from a survey battery administered at the end ${ }^{1}$ of the course consisting of (1) students goal orientation for the class; (2) students' future time perspective consisting of their ratings of the connectedness between their academic coursework and a STEM career and the perceived instrumentality of their specific course work for attaining STEM academic and career goals; and (3) students' emotional/affective reactions to the course. Students' metacognitive strategies and engagement were assessed with four scales from the Student Perceptions of Classroom Knowledge Building instrument (SPOCK) that assessed metacognitive self-regulation, knowledge building, question asking, and lack of engagement. Students' study time and study effort also were assessed. Profiles were derived using cluster analysis with SPSS. All instruments and procedures are fully described in Shell and Soh [16], Nelson et al. [11], and Flanigan et al. [6].

The students considered in our study provided many students in each engagement profile. The summary statistics for these profiles is provided in Table 2 .

1 The surveys could have also been conducted earlier in the course, providing the system with a priori student models.
Student behavior measures from their wiki data were based on the learning processes described in the Unified Learning Model (ULM) [14]. In the ULM, attention, repetition, and connection are the basic processes that underlie all learning. Attention is reflected in a simple page visit. Repetition and connection were operationalized as follows:

Connection: number of unique pages viewed/edited/commented

Repetition: average number of views/edits/comments per page (only considering pages the student interacts with).

\section{ANALYSIS}

Using the data described in the previous section, we now report the results of our investigation. For each research question, we first introduce our proposed hypothesis, followed by a description of the method employed to evaluate our hypothesis, and finally the results of our analysis.

\subsection{RQ1: Differences between Profiles}

Recall that our first research question (RQ1) asks whether CS1 students with different engagement profiles indeed behave differently in an online, wiki-based CSCL system. Based on the educational research surrounding student motivated engagement profiles, we hypothesize that:

H1: CS1 students with different engagement profiles will indeed behave differently in an online, wiki-based CSCL system.

That is, we hypothesize that student behaviors measured by connection and repetition (for the different action types: view, edit, and comment) will indeed differ between the engagement profiles. Summary statistics for these measures are provided in Table 3. We provide both the means, as well as the medians, due to (1) the non-normality of the data (determined using the Shapiro-Wilk test, which resulted in p-values all less than 0.001), and (2) the means are skewed due to the presence of outliers, especially at the larger end of the measures.

From Table 3, we observe that there do indeed appear to be differences between student behavior measures across the five engagement profiles in both the mean and median of the behaviors. To formally test for differences across the five engagement profiles, we employed the Kruskal-Wallis non-parametric one-way analysis of variance, rather than parametric ANOVA because of the non-normal empirical distributions of the measures. The null hypothesis of the Kruskal-Wallis tests is that each measure does not differ between the five engagement profiles. Rejecting this null hypothesis, instead, would confirm our research hypothesis $\mathrm{H} 1$.

We present the results of the Kruskal-Wallis tests in Table 4. From these results, we note that there were statistically significant differences between the five engagement profiles for several of the student behavior measures at 0.05 significance levels. In particular, students differed in: (1) how many times they viewed each wiki page that they visited (Repetition-View), (2) how many pages they edited (Connection-Edit), (3) how many pages they commented on (Connection-Comment), and (4) how many times they commented on each page (Repetition-Comment).

Thus, we reject the null hypothesis for the repetition measure for viewing behavior, connection measure for editing behavior, and both measures for commenting behavior. Instead, we confirm our hypothesis (H1) that CS1 student behaviors indeed do differ depending on student motivated engagement profiles in an online, wiki-based CSCL system. Therefore, considering student motivated engagement profiles can provide clues predicting different behaviors by CS1 students in a technology-mediated instruction or 
Table 3: Average Student Behavior Measures Across Engagement Profiles Note: Medians are provided in parenthesis due to non-normality and the skew of the measures caused by outliers

\begin{tabular}{c|r|c|c|c|c|c}
\multirow{2}{*}{ Measure } & Action & Strategic & Knowledge Builder & Surface & Apathetic & Learned Helpless \\
\cline { 3 - 7 } & View & $11.852(11)$ & $11.078(10)$ & $11.647(13)$ & $10.744(11)$ & $9.294(8.5)$ \\
\cline { 2 - 7 } Connection & Edit & $3.295(4)$ & $3.125(3)$ & $3.725(4)$ & $3.128(3)$ & $2.441(2)$ \\
\cline { 2 - 7 } & Comment & $2.852(3)$ & $2.797(3)$ & $3.059(3)$ & $2.641(2)$ & $1.824(1)$ \\
\hline \hline \multirow{3}{*}{ Repetition } & View & $6.475(5.8)$ & $7.146(7.798)$ & $7.506(6.729)$ & $6.084(5.273)$ & $5.256(4.059)$ \\
\cline { 2 - 7 } & Edit & $2.545(2)$ & $3.122(2.633)$ & $2.765(2.4)$ & $2.44(2.333)$ & $2.118(1.75)$ \\
\cline { 2 - 7 } & Comment & $2.46(2)$ & $3.077(2.5)$ & $2.645(2.5)$ & $2.326(2)$ & $1.709(1)$
\end{tabular}

Table 4: Results of the Kruskal-Wallis Tests Note: * indicates statistical significance at the 0.05 level

\begin{tabular}{c|r|c|c|c} 
Measure & \multicolumn{1}{|c|}{ Action } & \multicolumn{1}{c|}{$\boldsymbol{X}^{\mathbf{2}}$} & $\mathbf{p}$-value & Significance \\
\hline \hline \multirow{3}{*}{ Connection } & View & 4.08 & 0.3952 & \\
\cline { 2 - 5 } & Edit & 11.714 & 0.0196 & $*$ \\
\cline { 2 - 5 } & Comment & 11.257 & 0.0238 & $*$ \\
\hline \hline \multirow{3}{*}{ Repetition } & View & 11.484 & 0.0216 & $*$ \\
\cline { 2 - 5 } & Edit & 6.292 & 0.1784 & \\
\cline { 2 - 5 } & Comment & 11.303 & 0.0234 & $*$
\end{tabular}

e-learning environment, such as our creative thinking exercises performed within the online, wiki-based CSCL system.

\subsection{RQ2: Difference between Profile Predic- tions and Actual Behavior}

Now that we have observed that students with different engagement profiles do indeed differ in observed behaviors, we next turn to our related research question (RQ2) asking whether the differences match what is expected based on the differences between the profiles. That is, we want to know whether the educational research behind the engagement profiles indeed predicts how CS1 students will behave. We hypothesize that:

H2: The different behaviors exhibited by CS1 students in an online CSCL system will indeed match the behaviors that would be expected based on their motivated engagement profile.

In particular, based on the characteristics evidenced by CS1 students in each of the five profiles, we would expect that students in the strategic and knowledge builder profiles would have high levels of active learning processes that would be reflected in high connection and high repetition. Because of their focus on deep learning and interconnection of knowledge but less active engagement, knowledge builders would be expected to be somewhat higher in connection behaviors. Surface learners would be expected to be somewhat high in repetition as they are motivated to get good grades and achieve, but they would be expected to be lower in connection as they are not interested in deep learning. Apathetic students would be expected to be low in connection and repetition, if they did the exercise at all. Learned helpless students are harder to predict. They are motivated and report relatively high engagement and deep learning, but their distinguishing characteristic is that their learning efforts are not being successful. This dysfunction may not be identifiable from their overt behaviors so they may have reasonable levels of connection and repetition, but these may not be paying off in good learning. Conversely, as they lose motivation, they may begin to disengage and have lower levels of these.

To evaluate our hypothesis (H2) and answer our second research question, we compare the differences between the student behaviors across all six measure/action pairs, presented in Table 3. From the results, we make several important observations.
First, we consider the connection behaviors, which measure how many distinct pages students acted upon, and thus how broad their interactions were with the knowledge and exercise content stored in the wiki pages. We observe that students with strategic profiles had relatively high (mean and median) connection measures for all three actions (view, edit, comment), whereas students with apathetic and learned helpless profiles had some of the lowest connection measures. These behaviors match our expectations based on the educational research behind the profiles, described earlier in this subsection.

However, we also observe that contrary to our expectations, students with the surface profile had the highest connection measures across all three action types. Thus, it appears that surface learners attempted to maximize their utility (measured by course grades) by interacting with as many pages as possible.

Also contrary to our expectations, students with the knowledge builder profile had connection measures somewhere between the more connected (strategic and surface) and less connected (apathetic and learned helpless) profiles. Instead of our expectation that these knowledge builders would connect to a high number of pages, they appear to have instead behaved such that they connected only to the pages needed to build their desired knowledge and complete the exercises.

Second, we consider the repetition behaviors, which measure the average number of times students acted upon each page (considering only the pages they interacted with), and thus how actively engaged students were with the creative thinking exercises. This measures the depth of the students' interactions with the system. We observe again, for all three actions, that students with the strategic and surface profiles had some of the higher repetitions, whereas students with the apathetic and learned helpless profiles had some of the lowest repetitions. Again, this matches our expectations based on the definitions of the profiles, described above.

On the other hand, considering the repetition measures, we observe very different behavior for students with the knowledge building profile than we observed for the connection measures, in comparison to the other engagement profiles. In particular, knowledge builders had the highest (mean or median) repetition measures for all three actions. Consistent with their focus on deep learning, knowledge builders have higher engagement with the pages that they do interact with, possibly in an effort to maximize the learning from their interactions. Consistent with their emphasis on constructing knowledge rather than passively receiving it, we observe that the knowledge builders' higher repetition is greatest for the most intensive actions: editing and commenting on pages. Whereas viewing is a relatively passive action, editing and commenting on pages requires more thoughtfulness and active participation and contribute more to the advancement of group learning. Thus, knowledge builders contributed more to their collaborative creative thinking exercises than their peers. 
Combining these observations, we conclude that CS1 students indeed behaved similarly to what was predicted by their engagement profiles. Upon further reflection, differences in the observed behaviors of knowledge builder and surface learner profiles seem sensible given their differences in passive (viewing) and active (editing, commenting) behaviors. Thus, we have gained new insights into the engagement profiles themselves.

\section{DISCUSSION}

\subsection{Implications}

Summarizing the behaviors observed within each engagement profile, we draw the following key implications for each:

1. Strategic learners performed high levels of connection and repetition. Thus, strategic learners appear to attempt to perform a large number of interactions in order to achieve their learning goals, according to their motivation.

2. Knowledge builders performed moderate levels of connection, but high levels of repetition. Thus, knowledge builders appear to behave such that they learn efficiently, only connecting to as many pages as needed to learn and complete assignments, but maximizing their interactions with those pages to deeply learn them.

3. Surface learners perform opposite knowledge builders: they performed the highest levels of connection, but lower (comparatively) levels of repetition (especially for the more active actions: editing and commenting). Thus, surface learners appear to behave by performing more passive and less intensive actions than the students from other engagement profiles.

4. Apathetic learners performed low levels of connection and repetition, as expected. Thus, these students are unlikely to be actively engaged in technology-mediated instruction and elearning without prompting, scaffolding, or other engagement from the system itself.

5. Learned helpless learners performed the lowest levels of connection and repetition, indicating that in spite of their motivation, their struggles with learning caused them to give up, as predicted. Thus, like apathetic learners, they are unlikely to be actively engaged in technology-mediated instruction and elearning without prompting, scaffolding, or other engagement from the system itself. However, given their higher motivations, they might be more likely to respond to customization and other help from technology.

Based on our results and the above characterization of the differences in behaviors of CS1 students with different student motivated engagement profiles, we conclude that:

- CS1 students with different engagement profiles indeed do behave differently in (at least some) technology-mediated instruction and e-learning environments (e.g., an online, wikibased CSCL system)

- The engagement profiles themselves describe CS1 students such that their behavior within technology-mediated instruction and e-learning can be predicted.

Thus, we now have empirical evidence that student models constructed from educational research, such as the student motivated engagement profiles considered in this paper, can indeed be used to predict student behavior. Interestingly, although we only considered CS1 courses, our students were not just CS majors, and thus the results should generalize to a wide range of students taking CS1 courses.

Our results demonstrate that student models such as our engagement profiles could be used by technology (e.g., a wiki-based CSCL system) in order to anticipate student behavior before it occurs, then take intelligent actions to promote good behaviors and discourage, mitigate, or avoid unfruitful behaviors. For example, a system might encourage surface learners to spend more effort in their interactions, instead of performing a large number of less active interactions. Moreover, the system could encourage knowledge builders to teach their peers how to learn more efficiently and more in-depth. Finally, the system could provide customized, tailored support to help learned helpless students overcome their learning difficulties to make their high motivation more fruitful.

Furthermore, in cases where a system does not have an adaptive design, the predictable behaviors from engagement profiles (or other student models based on educational research) could still readily inform instructors during the semester to identify and seek out students with different profiles and perform remediation accordingly, which would be important in engaging and retaining increasingly diverse student populations in CS1 courses. Such information could also be used to form more effective teams for peer instruction and pair programming as well for CS1, for both majors and non-majors [13]. And in general, such information and knowledge helps us understand how students might respond to different assignments, helping plan activities that will benefit all students in the course, as well as prepare steps to help students become engaged with the course material and assignments to maximize learning.

Both of these benefits from student models are especially beneficial in a CS1 setting because (1) CS1 students are often new to college (i.e., freshman) and require guidance on how to learn, and not just in need of new course material, (2) introductory courses are often some of largest courses in terms of the number of students, making individualized support for students more difficult, especially if an instructor or software system must learn the student's characteristics, behaviors, and needs from scratch, and (3) CS1 courses are being increasingly taught with technologymediated instruction and e-learning environments, where instructor interactions with students are less active, and thus the technology used to support instruction plays an increasingly large role in the student's learning.

Finally, our results here have important implications on to other types of computer-mediated learner support. For example, Piech et al. [12] reported on how they modeled students arriving at their final solution in homework assignments, using machine learning techniques to autonomously create a graphical models progress through a homework assignment in an introductory programming course. Our work could inform their research with additional instructional design-i.e., incorporating threaded discussions and collaborative wikis - as well as enrich their modeling with our engagement profiles. Naps et al. [10] reported the use of visualization to illustrate various concepts in CS; Ericson et al. [5] reported the use of interactive features to engage students in an E-book; Sirkiä \& Sorva [17] reported the use of program visualizations of walking through lines of code and displaying results. Because of the primary media of these visualization is computer-based, our study has the potential of enriching these visualization approaches and helping researchers capture vital learner characteristics, thus enabling them to better understand and improve student learning in CS.

\subsection{Limitations}

Although our study has many promising implications for technology-mediated instruction and e-learning in CS1 (and possibly other) settings, we also note several limitations. 
First, our data only includes students who agreed to both take the student engagement profile survey, as well as allow us to track their activities as they used the wiki-based CSCL system. Although we had a large amount of participation, the students who agreed to participate might not have been representative of all students in the courses. We especially expect that lower motivation students (those likely to be in the apathetic or learned helpless profiles) would have been unlikely to participate. Indeed, within the lower performing profiles, our participants might have been skewed towards the more active students of these profile types, implying that these profiles might be expected to perform even fewer connection and repetition behaviors than we observed. Thus, our findings represent only a first look at how profiles might be used to help CS educators understand and intervene with these unmotivated students in technology-mediated and e-learning environments.

Furthermore, we have only considered one type of technologymediated instruction and e-learning environment: an online, wikibased CSCL system where students perform collaborative creative thinking exercises. It remains an open question how our results might relate to those in other educational settings (e.g., intelligent tutoring systems, distributed pair programming), which are interesting avenues for future work.

\section{CONCLUSIONS}

In conclusion, we have studied how student models determined by education research in the form of student motivated engagement profiles $[6,11,14,16]$ can be used to predict student behaviors in technology-mediated instruction and e-learning environments. Considering 7 CS1 courses with 249 students spanning 3 semesters, we investigated how students with different engagement profiles behaved in an online, wiki-based CSCL system while performing collaborative creative thinking exercises. We discovered that not only do students of different profiles behave differently (with respect to how students interact with the exercises and technology), but their behaviors were generally as expected based on the descriptions of the profiles used as student models. As a result, we now have evidence that student models (that could be matched a priori before students interact with the technology or assignment based on separate data from system usage) can be used to predict student behavior, and thus could be useful inputs to inform and guide customized educational support tailored to individual student's needs within the e-learning technology.

In the future, we intend to (1) further explore what other types of student behaviors might also be predictable using educational research-informed student models, including in other types of elearning environments, (2) develop tools for customizing student support exploiting such models, and (3) evaluate the effectiveness of such tools.

\section{ACKNOWLEDGMENTS}

This material is based upon work supported by the National Science Foundation under grants no. 1122956 and 1431874. Additional support was provided by a UNL Phase II Pathways to Interdisciplinary Research Centers grant.

\section{REFERENCES}

[1] Cooper, S., Nam, Y.J., \& Si, L. 2012. Initial Results of Using an Intelligent Tutoring System with Alice. In Proc. of ITiCSE'12, 138-143.

[2] Cooper, S. \& Sahami, M. 2013. Reflections on Stanford's MOOCs, Communications of the ACM, 56(2):28-30.
[3] Desmarais, M. C. \& Baker, R. S. 2012. A Review of Recent Advances in Learner and Skill Modeling in Intelligent Learning Environments. Journal of User Modeling and UserAdapted Interaction, 22(1-2):9-38.

[4] Eck, A., Soh, L.-K., \& Brassil, C. 2013. Supporting Active Wiki-based Collaboration. In. Proc. of CSCL'13, 176-183.

[5] Ericson, B. J., Guzdial, M. J., \& Morrison, B. B. 2015. Analysis of Interactive Features Designed to Enhance Learning in an Ebook. In Proc. of ICER'2015, 169-178.

[6] Flanigan, A. E., Peteranetz, M. S., Shell, D. F., \& Soh, L.K. 2015. Exploring Changes in Computer Science Students' Implicit Theories of Intelligence Across the Semester. In Proc. of ICER'15, 161-168.

[7] Jovanovic, M., Vukicevic, M., \& Milovanovic, M. 2012. Using Data Mining on Student Behavior and Cognitive Style Data for Improving E-Learning Systems: A Case Study, Int. Jour. of Computational Intelligence Systems, 5(3):597-610.

[8] Khandaker, N. \& Soh, L.-K. 2010. ClassroomWiki: A Collaborative Wiki for Instructional Use with Multiagent Group Formation, IEEE Trans. Learn. Technologies, 3(3):190-202.

[9] Miller, L.D. \& Soh, L.-K. 2013. Meta-Reasoning Algorithms for Improving Analysis of Student Interactions with Learning Objects using Supervised Learning. In Proc. of EDM'2013, 129-136.

[10] Naps, T. L, et al. 2003. Exploring the Role of Visualization and Engagement in Computer Science Education. $A C M$ SIGCSE Bulletin, 35(2):131-152.

[11] Nelson, K.G., et al. 2015. Motivational and Self-Regulated Learning Profiles of Students Taking a Foundational Engineering Course. J. Eng. Educ. 104(1):74-100.

[12] Piech, C., et al. 2012. Modeling How Students Learn to Program. In Proc. of SIGCSE'2012, 153-160.

[13] Porter, L., Guzdial, M., Mcdowell, C., \& Simon, B. 2013. Success in Introductory Programming: What Works? $C A C M$, 56(8):34-36.

[14] Shell, D.F., et al. 2010. The Unified Learning Model: How Motivational, Cognitive, and Neurobiological Sciences Inform Best Teaching Practices. Springer, New York.

[15] Shell, D.F. \& Husman, J. 2008. Control, Motivation, Affect, and Strategic Self-regulation in the College Classroom: A Multidimensional Phenomenon. J. Educ. Psychol. 100(2): 443-459.

[16] Shell, D.F. \& Soh, L.K. 2013. Profiles of Motivated SelfRegulation in College Computer Science Courses: Differences in Major Versus Required Non-major Courses. J. Sci. Educ. Technol., 22:899-913.

[17] Sirkiä, T., \& Sorva, J. 2015. How Do Students Use Program Visualizations within an Interactive Ebook? In Proc. of ICER'2015, 179-188.

[18] Soh, L.-K., Shell, D. F., Ingraham, E., Ramsay, S., \& Moore, B. 2015. Viewpoint: Improving Learning and Achievement in Introductory Computer Science through Computational Creativity. CACM, 58(8):33-35.

[19] Wiggins, J. B., et al. 2015. JavaTutor: An Intelligent Tutoring System that Adapts to Cognitive and Affective States during Computer Programming. In Proc. of SIGCSE'2015, 599. 